\title{
Topology optimization of grating couplers for the efficient excitation of surface plasmons
}

\author{
Andkjær, Jacob Anders; Sigmund, Ole; Nishiwaki, Shinji; Nomura, Tsuyoshi
}

Published in:

Optical Society of America. Journal B: Optical Physics

Link to article, DOI:

10.1364/JOSAB.27.001828

Publication date:

2010

Document Version

Publisher's PDF, also known as Version of record

Link back to DTU Orbit

Citation (APA):

Andkjær, J. A., Sigmund, O., Nishiwaki, S., \& Nomura, T. (2010). Topology optimization of grating couplers for the efficient excitation of surface plasmons. Optical Society of America. Journal B: Optical Physics, 27(9), 18281832. https://doi.org/10.1364/JOSAB.27.001828

\section{General rights}

Copyright and moral rights for the publications made accessible in the public portal are retained by the authors and/or other copyright owners and it is a condition of accessing publications that users recognise and abide by the legal requirements associated with these rights.

- Users may download and print one copy of any publication from the public portal for the purpose of private study or research.

- You may not further distribute the material or use it for any profit-making activity or commercial gain

- You may freely distribute the URL identifying the publication in the public portal 


\title{
Topology optimization of grating couplers for the efficient excitation of surface plasmons
}

\author{
Jacob Andkjær, ${ }^{1, *}$ Shinji Nishiwaki, ${ }^{2}$ Tsuyoshi Nomura, ${ }^{3}$ and Ole Sigmund ${ }^{1}$ \\ ${ }^{1}$ Department of Mechanical Engineering, Technical University of Denmark, Nils Koppels Alle, Building 404, \\ 2800 Kgs. Lyngby, Denmark \\ ${ }^{2}$ Department of Mechanical Engineering and Science, Kyoto University, Kyoto 606-8501, Japan \\ ${ }^{3}$ Toyota Central R\&D Laboratories Inc., Aichi 480-1192, Japan \\ *Corresponding author: jban@mek.dtu.dk
}

Received June 15, 2010; accepted June 26, 2010;

posted July 7, 2010 (Doc. ID 130211); published August 18, 2010

\begin{abstract}
We propose a methodology for a systematic design of grating couplers for efficient excitation of surface plasmons at metal-dielectric interfaces. The methodology is based on a two-dimensional topology optimization formulation based on the $H$-polarized scalar Helmholtz equation and finite-element method simulations. The efficiency of the method is demonstrated by optimized designs for input and output grating couplers for an $\mathrm{Ag}-\mathrm{SiO}_{2}$ interface. The results indicate that slanted grove gratings may raise the coupling efficiency above $68 \%$ where the highest previously reported value was $50 \%$. (C) 2010 Optical Society of America

OCIS codes: 240.6680, 050.1590, 050.2770.
\end{abstract}

\section{INTRODUCTION}

Surface plasmons are electromagnetic waves trapped at the interface between a metal with a negative real part and a dielectric with a positive real part of the permittivity [1]. Surface plasmonic effects may be used in the realization of pure photonic circuits which potentially will be much faster than electronic circuits, in the lighting industries for light-emitting diodes or organic light-emitting diodes [2,3] and in solar cells [4]. Common for all applications is the problem of efficient coupling of light into the surface plasmon or vice versa. For more detailed overviews of surface plasmonic effects see $[5,6]$.

Surface plasmons can be excited by electrons or photons. In this work we use photons in the form of a light beam directed toward the interface between a dielectric material and a metal. The incoming photons are scattered and transfer momentum to the nearly free electrons of the metals. With the right coupling, the nearly free electrons of the conductor will collectively oscillate in resonance with the incoming beam. This causes the light to be trapped at the interface and form a plasma oscillation in the optical frequency range. Figure 1 shows the theoretically predicted electromagnetic field for a surface plasmon. The nearly free electrons oscillate in resonance with the incoming light and the "+" and "-" regions represent regions with low and high surface charge densities, respectively. The magnitude of the electric field $E$ is higher in the dielectric than in the metal due to the energy dissipation in the metal. The electric field oscillates in the $(x, y)$-plane making the surface plasmons transverse magnetic in character; hence the magnetic field $H_{z}$ is given in the $z$-direction.

Efficient coupling of light into the interface is crucial. Common methods for coupling light into a surface plasmon are based on prism couplers (e.g., Kretschmann and Otto configuration) or grating couplers [1]. On small scales the use of a prism is impractical, which motivates the use of grating couplers. A grating coupler comprises a topology that enables a wave vector match between the incident light and the surface plasmon, without the use of a prism. The topology of these grating couplers can be designed in many ways and the design has a big influence on the efficiency of the coupling. This leads to the use of optimization techniques for finding the most optimal design. Three decades ago Tamir and Peng [7] performed analytical as well as simple numerical studies on the effect of different groove designs on the coupling efficiency. Other contributions have been presented in [8-10], and lately Lu et al. [11] performed a systematic optimization of coupling into a surface plasmon. Using a hierarchal search optimization algorithm, varying the width and position of 14 grooves the authors achieved an excitation efficiency of $50 \%$, which is the highest efficiency reported in the literature for a grating coupler. In the present paper we extend the work of $\mathrm{Lu}$ et al. [11] to allow free shapes and topologies of the groves and we study not only the case of coupling into the plasmon but also the coupling of light from the plasmon into the dielectric surroundings. By increasing the design freedom using the topology optimization method we manage to improve the coupling efficiency from $50 \%$ reported by Lu et al. [11] to more than $68 \%$ which is obtained for a coupling topology with slanted groves.

Topology optimization is a computational tool that optimizes material distribution for a given objective function with geometrical and/or physical constraints [12]. The concept was introduced more than two decades ago in [13] for structural mechanics, but the method has later been used to tailor new materials or mechanisms in various fields of engineering such as fluid dynamics (e.g., [14]), micro-electro-mechanical systems (e.g., [15]), photonics (e.g., [16]), and multiphysics problems (e.g., [17]). Us- 


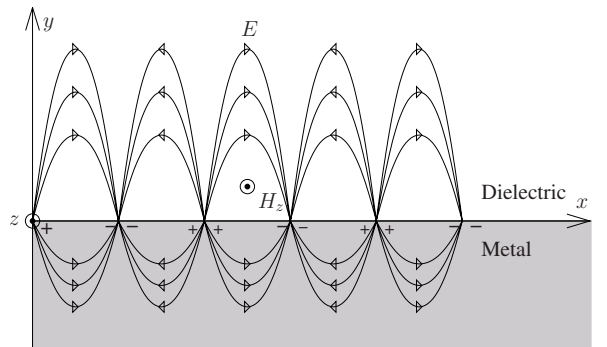

Fig. 1. A surface plasmon induced in the interface between a metal and a dielectric. + and - signs represent regions with low and high surface charge densities, respectively. The electric field $E$ oscillates in the $(x, y)$-plane making the surface plasmons transverse magnetic in character (hence magnetic field $H_{z}$ in the $z$-direction).

ing topology optimization it is possible to find solutions that are not easily found by intuition, such as materials with negative Poisson's ratio [18] and negative thermal expansion coefficient [19]. Thus topology optimization is a suitable method to use in order to design grating couplers for the efficient excitation of surface plasmons.

The layout of the paper is as follows. Section 2 summarizes the theory behind modeling topology optimization of surface plasmonic problems. Optimized designs of grating couplers are presented in Section 3 and conclusions are given in Section 4.

\section{TOPOLOGY OPTIMIZATION OF TWO- DIMENSIONAL SURFACE PLASMON GRATING COUPLERS}

The implementation of the two-dimensional topology optimization problem is based on the finite-element method using a combination of the commercial programs COMSOL 3.4 and MATLAB 7.5.0 (R2007b). We use the material and geometry settings from [11]. The computational models for the input and output couplers are presented in Figs. 2(a) and 2(b), respectively. Both models consist of a $\mathrm{SiO}_{2}$-domain, $\Omega_{\mathrm{SiO}_{2}}$; an Ag-domain, $\Omega_{\mathrm{Ag}}$; and a design domain, $\Omega_{\text {des }}$, where $\mathrm{SiO}$ and $\mathrm{Ag}$ shall be distributed freely. The design domain $\Omega_{\text {des }}$ has a width and height of $2.5 \mu \mathrm{m}$ and $50 \mathrm{~nm}$, respectively. For the input coupler a timeharmonic wave is excited at $\Gamma_{\text {inp }}$ with a free-space wavelength of $476 \mathrm{~nm}$. The wave is Gaussian shaped with a

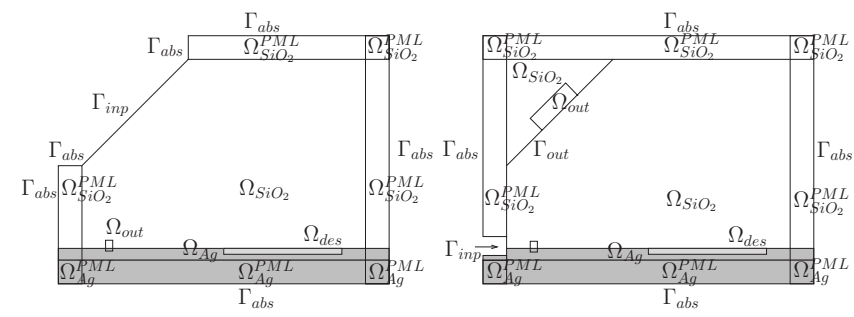

(a) Input coupler

(b) Output coupler

Fig. 2. Computational models for the input and output couplers containing a dielectric $\left(\Omega_{\mathrm{SiO}_{2}}\right)$, a metal $\left(\Omega_{\mathrm{Ag}}\right)$, and a design domain $\left(\Omega_{\mathrm{des}}\right)$. A plane wave is excited at $\Gamma_{\mathrm{inp}}$. A surface plasmon will be induced in the interface between the two media and the energy flux is measured in the domain $\Omega_{\text {out }}$. To avoid reflections from the boundaries, PML domains $\left(\Omega^{\mathrm{PML}}\right)$ and absorbing boundary condition $\Gamma_{\text {abs }}$ have been introduced. All interior boundaries have continuous boundary condition. full width at half-maximum $(\mathrm{FWHM})=1 \mu \mathrm{m}$ and has an incident angle of $45^{\circ}$. For the output grating coupler the surface plasmon is excited at $\Gamma_{\text {inp }}$ with an exponentially shaped electromagnetic wave matching the skin depth. For both cases, the permittivities of $\mathrm{SiO}_{2}$ and $\mathrm{Ag}$ corresponding to the given wavelength are $\epsilon_{r}=2.25$ and $\epsilon_{r}$ $=-7.06-i 0.27$, respectively, and the permeability is assumed to be $\mu_{r}=1$ for both materials. For the input coupler the incident beam will induce a surface plasmon at the $\mathrm{Ag}-\mathrm{SiO}_{2}$ interface when the wave vectors match due to the grating coupler and vice versa for the output coupler. The power flux of the surface plasmon is measured using the averaged Poynting vector in the domain $\Omega_{\text {out }}$. Reflections from the boundaries are eliminated using perfectly matched layers (PMLs) [20]. Furthermore, absorbing boundary conditions $\Gamma_{\text {abs }}$ have been introduced on all outer boundaries.

\section{A. Governing Equation}

The wave propagation problem is governed by the $H$-polarized scalar Helmholtz equation,

$$
\begin{gathered}
\nabla \cdot\left(\epsilon_{r}^{-1} \nabla H_{z}(\mathbf{r})\right)+k_{0}^{2} H_{z}(\mathbf{r})=0 \quad \text { in } \Omega \\
\frac{\partial}{\partial x}\left(\frac{s_{y}}{s_{x}} \epsilon_{r}^{-1} \frac{\partial H_{z}(\mathbf{r})}{\partial x}\right)+\frac{\partial}{\partial y}\left(\frac{s_{x}}{s_{y}} \epsilon_{r}^{-1} \frac{\partial H_{z}(\mathbf{r})}{\partial y}\right)+s_{x} s_{y} k_{0}^{2} H_{z}(\mathbf{r}) \\
=0 \quad \text { in } \Omega^{\mathrm{PML}}
\end{gathered}
$$

where $k_{0}=\omega / c$ is the free-space wave number, $\omega$ is the angular frequency, $c$ is the speed of light in vacuum, $H_{z}(\mathbf{r})$ is the unknown magnetic field in the plane $\mathbf{r}=(x, y)$, and $s_{x}$ and $s_{y}$ are complex functions of the position and govern the damping properties of the PML.

The boundary conditions are specified for both problems as

$$
\begin{gathered}
\mathbf{n} \cdot\left(\epsilon_{r}^{-1} \nabla H_{z}(\mathbf{r})\right)=0 \quad \text { on } \Gamma_{\text {con }}, \\
\mathbf{n} \cdot\left(\epsilon_{r}^{-1} \nabla H_{z}(\mathbf{r})\right)+i k_{0} \sqrt{\epsilon_{r}^{-1}} H_{z}=0 \text { on } \Gamma_{\mathrm{abs}},
\end{gathered}
$$

where $i=\sqrt{-1}$ and $\mathbf{n}$ is the outward-pointing normal vector. The boundary condition for the input coupler with the Gaussian shaped beam is

$$
\begin{aligned}
& \mathbf{n} \cdot\left(\boldsymbol{\epsilon}_{r}^{-1} \nabla H_{z}(\mathbf{r})\right) \\
& \quad=i k_{0} \sqrt{\boldsymbol{\epsilon}_{r}^{-1}}\left[2 \exp \left(-\frac{\left(x-x_{0}\right)^{2}}{2\left(\frac{1}{\sqrt{2}} \sigma\right)^{2}}\right) H_{0}-H_{z}\right] \text { on } \Gamma_{\text {inp }},
\end{aligned}
$$

where $x_{0}$ is the $x$-coordinate at the center of $\Gamma_{\text {inp }}, \sigma$ $=\mathrm{FWHM} /[2 \sqrt{2 \log 2}]$, and $H_{0}$ is the incident magnetic field amplitude. For the output coupler the boundary condition is modeled with an exponentially shaped field, using knowledge of the skin depth and the vertical wave vector $k_{y, \mathrm{Ag}}$ in the silver, 


$$
\begin{gathered}
\mathbf{n} \cdot\left(\epsilon_{r}^{-1} \nabla H_{z}(\mathbf{r})\right)=i k_{0} \sqrt{\epsilon_{r}^{-1}}\left[2 \exp \left(-\left|k_{y, \mathrm{Ag}}\right|\left|y_{0}-y\right|\right) H_{0}\right. \\
\left.-H_{z}\right] \quad \text { on } \Gamma_{\mathrm{inp}},
\end{gathered}
$$

where $k_{y, \mathrm{Ag}}$ is found analytically to be $13.5 \times 10^{6} \mathrm{~m}^{-1}$ and $y_{0}$ is the $y$-coordinate of the interface.

\section{B. Design Variables, Material Interpolation, and Design Field}

Following the standard topology optimization approach, the material distribution in the design domain $\Omega_{\text {des }}$ can be changed by varying the permittivity of individual finite elements continuously between the material values for $\mathrm{SiO}_{2}$ and $\mathrm{Ag}$. The design variables are relative element material densities $\rho_{e} \in[0 ; 1]$, where $\rho_{e}=0$ corresponds to $\mathrm{Ag}$ and $\rho_{e}=1$ corresponds to $\mathrm{SiO}_{2}$. The following interpolation models the relation between element densities and element permittivities:

$$
\begin{aligned}
\epsilon_{r}\left(\rho_{e}\right)= & \epsilon_{\mathrm{Ag}}^{\prime}+\rho_{e}\left(\epsilon_{\mathrm{SiO}_{2}}^{\prime}-\epsilon_{\mathrm{Ag}}^{\prime}\right)-i\left[\epsilon_{\mathrm{Ag}}^{\prime \prime}+\rho_{e}\left(\epsilon_{\mathrm{SiO}_{2}}^{\prime \prime}-\epsilon_{\mathrm{Ag}}^{\prime \prime}\right)\right] \\
& +i 4 d\left(\rho_{e}^{2}-\rho_{e}\right),
\end{aligned}
$$

where ' and ", respectively, denote the real and the imaginary parts of the permittivity for the given material and $d$ is a factor introducing artificial damping. This damping induces an energy loss for intermediate values of $\rho$ and disfavors non-discrete (0/1) values in the final design [21]. Remark that the continuous design variables are introduced in order to be able to use efficient gradientbased math-programming methods for solving the optimization problem, in turn assuring convergence within a couple of hundred finite-element analyses.

To ensure manufacturable and mesh independent designs we use image processing-based filtering techniques. An overview of different filtering techniques is presented in [22] but in this work we use the latest Guest filter [23] which ensures a minimum length scale for both the silica and the silver phases. This means that regions with silica or silver cannot become smaller than the chosen filter size. Thus the designs can be tailored to meet manufacturing criteria. We denote the filtered design variables by $\widetilde{\rho}_{e}$.

\section{Objective Function and Optimization Problem}

The goal of the optimization problem is to maximize the averaged Poynting vector (power flow) in $\Omega_{\text {out }}$ in the negative direction of the $x$-axis, $\mathbf{n}=(-1,0)$, for the input coupler and in the beam direction, $\mathbf{n}=(-\sqrt{2} / 2, \sqrt{2} / 2)$, for the output coupler. The gradient-based optimization routine method of moving asymptotes (MMA) [24] is applied to update the design variables in an iterative approach until convergence. A volume constraint is added to the optimization problem, not because we want to limit the usage of one material, but to avoid excess islands of material and ensure a cleaner final design. Thus the optimization problem is formulated as

$$
\max _{\rho} \Phi\left(H_{z}(\widetilde{\rho})\right)=P_{\text {out }}=\int_{\Omega_{\text {out }}} \frac{1}{2 L_{x} \epsilon_{0} \omega} \Re\left(i \epsilon_{r}^{-1} H_{z}^{*} \nabla H_{z}\right) \cdot \mathbf{n} \mathrm{d} \mathbf{r},
$$

s.t. Helmholtz equation

Boundary conditions

$$
\frac{1}{V_{\Omega_{\mathrm{des}}}} \int_{\Omega_{\mathrm{des}}} \tilde{\rho}(\mathbf{r}) \mathrm{d} \mathbf{r}-\beta \leq 0, \quad 0 \leq \rho(\mathbf{r}) \leq 1,
$$

where * denotes complex conjugate, $P_{\text {out }}$ is the averaged power flow through $\Omega_{\text {out }}, L_{x}$ is the width of $\Omega_{\text {out }}, \mathbf{n}$ gives the direction of the power flux, $\Re\left(\right.$ ) is the real part, $V_{\Omega_{\text {des }}}$ is the total volume of $\Omega_{\mathrm{des}}$, and $\beta$ is the maximum allowed volume fraction of $\mathrm{SiO}_{2}$.

\section{Efficiency}

The decay length of the surface plasmon and its skin depth in the two materials are used to evaluate the power flux of the surface plasmon perpendicular to the interface at the first groove of the grating. The electric field of the surface plasmon along the interface decreases with the distance $|x|$ to the grating as $\exp \left(-2 k_{x, \mathrm{SP}}^{\prime \prime}|x|\right)$, where $k_{x, \mathrm{SP}}^{\prime \prime}$ is the imaginary part of the surface plasmon wave vector along the interface and can be derived analytically to $k_{x, \mathrm{SP}}^{\prime \prime}=214.6 \times 10^{3} \mathrm{~m}^{-1}$ for this problem. At the decay length, $\delta_{\mathrm{SP}}=2.33 \times 10^{-6} \mathrm{~m}$, the electric field has decreased by a factor of $\exp (-1)$. Thus the horizontal center of the output domain $\Omega_{\text {out }}$ is positioned exactly at the decay length of the surface plasmon, and hence $P_{\text {out }}$ should be divided by the factor $\exp (-1)$ in order to find the power flow at the first groove of the grating. Likewise the electric field of the surface plasmon normal to the interface decays with the distance $|y|$ as $\exp \left(-\left|k_{y}\right||y|\right)$, where $k_{y}$ is the real part of the surface plasmon wave vector perpendicular to the interface. The wave vector is dependent on the material in which it propagates; thus the wave vectors are analytically found to be $k_{y, \mathrm{Ag}}=42.5 \times 10^{6} \mathrm{~m}^{-1}$ and $k_{y, \mathrm{SiO}_{2}}=13.5 \times 10^{6} \mathrm{~m}^{-1}$, and thus the skin depths become $\delta_{\mathrm{Ag}}=23.5 \times 10^{-9} \mathrm{~m}$ and $\delta_{\mathrm{SiO}_{2}}=73.9 \times 10^{-9} \mathrm{~m}$. The height of the output domain $\Omega_{\text {out }}$ is set to the skin depth in the two materials, and thus the power flow through an infinite line perpendicular to the interface can be found as $P_{\text {out }} /[1-\exp (-1)]$. For the input coupler the efficiency of the coupling is found as the power flux of the surface plasmon at the first groove of the grating divided by the power flux at the input boundary $\Gamma_{\text {inp }}$,

$$
\text { Efficiency }=\frac{P_{\text {out }}}{\exp (-1)[1-\exp (-1)] P_{\text {inp }}} .
$$

To avoid reflections from the incident beam on the silver surface, the power flow at the input, $P_{\text {inp }}$, boundary is found when all domains are set to silica.

In the reverse case (output coupler) the efficiency is found as the power flow through the line parallel to the output domain $\Omega_{\text {out }}$ divided by the power flux of the surface plasmon at the first groove of the grating. In order to find the power flow of the surface plasmon at the first grating, we use the power flow at the input boundary and the distance $L_{\mathrm{SP}}$ from the boundary to the first grating to calculate the decrease in the electric field due to energy dissipation along the interface,

$$
\text { Efficiency }=\frac{P_{\text {out }}}{\exp \left(-2 k_{x, \mathrm{SP}}^{\prime \prime} L_{\mathrm{SP}}\right) P_{\text {inp }}},
$$

where $P_{\text {inp }}$ and $P_{\text {out }}$ are evaluated at $\Gamma_{\text {inp }}$ and $\Gamma_{\text {out }}$, respectively. 


\section{E. Discretization}

The modeling problem is solved using the finite-element method; thus the discretized set of equations can be written in the form

$$
\mathbf{S}(\rho) \mathbf{u}=\mathbf{f}
$$

where $\mathbf{S}(\rho)$ is the system matrix (which directly depends on the density variables), $\mathbf{u}$ is the state field, and $\mathbf{f}$ is the load vector. The design domain $\Omega_{\text {des }}$ is discretized using 500.000 bi-linear quadrilateral elements and the rest of the domains are discretized using triangular linear elements with a maximum length of $25 \mathrm{~nm}$. This yields a total of 680.842 elements in the input coupler case and 696.172 elements in the output coupler case, and the design elements have a side length of $0.5 \mathrm{~nm}$. This rather fine resolution is not strictly required from convergence considerations; however, we have chosen this fine discretization in order to be able to reproduce the grating coupler from [11] using a regular element division. The state field $\mathbf{u}$ is given by continuous elements, whereas the design field is modeled using piecewise constant elements in order to obtain sharp material boundaries at interfaces.

\section{F. Sensitivity Analysis}

The adjoint method is used in order to find the sensitivities for the gradient-based optimization algorithm, MMA. The sensitivities for a discretized problem in the form of Eq. (11) with the objective function $\Phi$ are given in [21] as

$$
\frac{\mathrm{d} \Phi}{\mathrm{d} \widetilde{\rho}_{e}}=\frac{\partial \Phi}{\partial \widetilde{\rho}_{e}}+2 \Re\left[\boldsymbol{\lambda}^{T}\left(\frac{\partial \mathbf{S}}{\partial \widetilde{\rho}_{e}} \mathbf{u}-\frac{\partial \mathbf{f}}{\partial \widetilde{\rho}_{e}}\right)\right],
$$

where the adjoint vector $\boldsymbol{\lambda}$ is obtained from the solution of the adjoint problem

$$
\mathbf{S}^{T} \boldsymbol{\lambda}=-\frac{\partial \Phi}{\partial \mathbf{u}}
$$

The rightmost term of Eq. (12) is zero due to the design domain not being a part of the output domain. The terms $\partial \mathbf{S} / \partial \widetilde{\rho}_{e}$ and $\partial \mathbf{f} / \partial \widetilde{\rho}_{e}$ are known as part of the direct solution of Eq. (11). For the present finite-element problem the system matrix $\mathbf{S}$ is symmetric and hence the solution of the adjoint problem in Eq. (13) can be performed very efficiently by the reuse of the factorization of $\mathbf{S}$ computed for the direct analysis stated in Eq. (11). To summarize, this means that the computational costs associated with obtaining the sensitivities is ignorable compared to the solution of the original problem in Eq. (11). For simplification we use the notation $A=1 /\left(2 L_{x} \epsilon_{0} \omega\right) \Re\left(i \epsilon_{r}^{-1} \mathbf{u}^{*} \nabla \mathbf{u}\right) \cdot \mathbf{n}$. Thus the derivatives with respect to the state variables, $\partial \Phi / \partial \mathbf{u}$, can then be found by the chain rule to be

$$
\frac{\partial \Phi}{\partial \mathbf{u}}=\int_{\Omega_{\text {out }}}\left(\frac{\partial A}{\partial \mathbf{u}}+\frac{\partial A}{\partial \nabla \mathbf{u}} \cdot \nabla\right) \mathrm{d} \mathbf{r},
$$

where

$$
\frac{\partial A}{\partial \mathbf{u}}=\frac{-1}{4 L_{x} \epsilon_{0} \omega} i \epsilon_{r}^{*-1} \nabla \mathbf{u}^{*} \cdot \mathbf{n},
$$

$$
\frac{\partial A}{\partial \nabla \mathbf{u}}=\frac{1}{4 L_{x} \epsilon_{0} \omega} i \epsilon_{r}^{-1} \mathbf{u}^{*} \mathbf{n} .
$$

\section{G. Algorithm}

The overall optimization algorithm is described in a simplified pseudocode in [22]. We stop the optimization when the maximum difference of the densities in two iterations is less than $1 \%$ or 1000 iterations are reached. The regularization filter [23] will lead to designs with a small transition area of intermediate densities. Such designs are non-realizable; hence all densities lower than 0.9 are set to zero and the rest of the densities are set to 1 after convergence, in order to obtain discrete (0/1) designs. This step also ensures that the artificial damping, introduced in Eq. (7), does not contribute to the final computation of the problem in order to get the final efficiency.

\section{RESULTS}

We use an initial guess of pure silver in the design domain $\Omega_{\text {des }}$, which is shown in Fig. 3(a) with length scales. For both input and output coupler cases we impose a volume fraction constraint on the silica of 0.3 and the minimum length scales are set to $5 \mathrm{~nm}$ for both material phases. The optimized designs for the input and output couplers obtained using the described methodology are presented in Figs. 3(b) and 3(c), respectively. For the purpose of comparison we have presented the grating coupler from [11] in Fig. 3(d).

The optimized design for the input coupler consists of 13 inclined grooves of increasing width and decreasing depth. The optimized design represents an efficiency of $68.7 \%$ which is a approximately a factor of 0.4 higher than the efficiency of the grating coupler from [11]. The optimized design of the output coupler is very similar to the optimized design of the input coupler. The output coupler has an extra groove at the right side of the design domain resulting in a total of 14 inclining grooves, the same number as the grating coupler from [11]. The 14th groove is also found in the design of the input coupler at intermediate steps in the optimization loop, but it disappears in later steps. The manufacturability of the optimized designs may be ensured by choosing appropriate minimum length scales. The designs presented here are shown as specific solutions; however, the general concept of the improved efficiency by inclined grooves may inspire future realizations of efficient grating couplers. Figure 4 shows the absolute magnetic fields of the optimized designs for

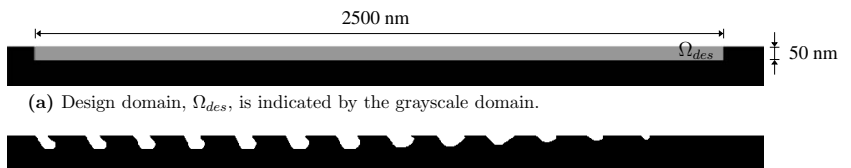

(b) Input coupler, efficiency: $68.71 \%$

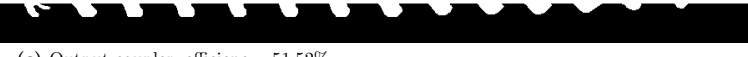

(c) Output coupler, efficiency: $51.52 \%$

12 - 12

(d) Coupler from [11], efficiency: $49.74 \%$

Fig. 3. Optimized designs 


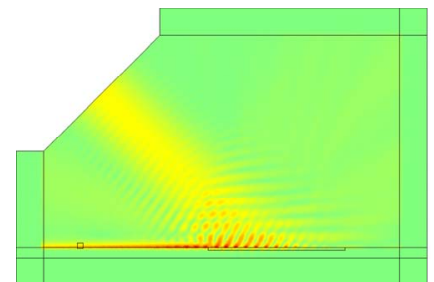

(a) Input coupler

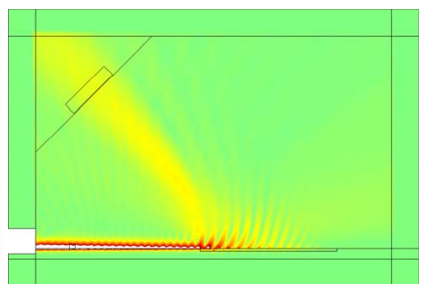

(b) Output coupler
Fig. 4. (Color online) FE simulations showing the absolute magnetic field for the optimized designs.

the input and output couplers. At the end we note that the optimized output coupler is indeed better than the reversed input coupler. Equal topologies for the two cases are not expected due to different loss mechanisms in the incoming and outgoing wave cases.

\section{CONCLUSION}

This paper proves that the topology optimization method can be applied to the design of nano-photonic grating couplers. Results indicate that efficiencies beyond $68 \%$ are possible for slanted grove-based gratings. The highest reported efficiency for vertical groves is $50 \%$. The big improvement may warrant the development of improved manufacturing technologies that allow slanted groves. Future work may include topology optimization of grating couplers for frequency ranges and variable input wave directions, which will ensure more robust but probably less efficient gratings.

\section{ACKNOWLEDGMENTS}

This work is supported from a Eurohorcs/ESF European Young Investigator Award [25] through the grant "Synthesis and topology optimization of optomechanical systems" and from the Danish Research Council for Technology and Production through the grant "Topology Optimization of Electromagnetic Metamaterials for Miniaturization of Wireless Communication Antennas" [26]. Fruitful discussions with members of the TopOpt-group [27] are also gratefully acknowledged.

\section{REFERENCES}

1. H. Raether, Surface Plasmons on Smooth and Rough Surfaces and on Gratings (Springer-Verlag, 1988).

2. W. L. Barnes, "Turning the tables on surface plasmons," Nature Mater. 3, 588-589 (2004).

3. P. A. Hobson, J. A. E. Wasey, I. Sage, and W. L. Barnes, "The role of surface plasmons in organic light-emitting diodes," IEEE J. Quantum Electron. 8, 378-386 (2002).

4. V. E. Ferry, L. A. Sweatlock, D. Pacifici, and H. A. Atwater, "Plasmonic nanostructure design for efficient light coupling into solar cells," Nano Lett. 8, 4391-4397 (2008).
5. H. A. Atwater, "The promise of plasmonics," Sci. Am. 296, 56-63 (2007).

6. W. L. Barnes, A. Dereux, and T. W. Ebbesen, "Surface plasmon subwavelength optics," Nature 424, 824-830 (2003).

7. T. Tamir and S. T. Peng, "Analysis and design of grating couplers," Appl. Phys. 14, 235-254 (1977).

8. C. Peng and W. A. Challener, "Input-grating couplers for narrow Gaussian beam: influence of groove depth," Opt. Express 12, 6481-6490 (2004).

9. G. Leveque and O. J. F. Martin, "Numerical study and optimization of a diffraction grating for surface plasmon excitation,” Proc. SPIE 5927, 246-254 (2005).

10. Y. Huang, "Optimal design for the grating coupler of surface plasmons," in Proceedings of the COMSOL Conference (2008), 4980 http://www.comsol.com/papers/4980.

11. J. Lu, C. Petre, E. Yablonovitch, and J. Conway, "Numerical optimization of a grating coupler for the efficient excitation of surface plasmons at an $\mathrm{Ag}-\mathrm{SiO}_{2}$ interface," J. Opt. Soc. Am. B 24, 2268-2272 (2007).

12. M. P. Bendsøe and O. Sigmund, Topology Optimization: Theory, Methods and Applications (Springer-Verlag, 2003).

13. M. P. Bendsøe and N. Kikuchi, "Generating optimal topologies in optimal design using a homogenization method," Comput. Methods Appl. Mech. Eng. 71, 197-224 (1988).

14. T. Borrvall and J. Petersson, "Topology optimization of fluids in Stokes flow," Int. J. Numer. Methods Fluids 41, 77107 (2003).

15. J. Jonsmann, O. Sigmund, and S. Bouwstra, "Compliant thermal microactuators," Sens. Actuators, A 76, 463-469 (1999).

16. P. I. Borel, A. Harpøth, L. H. Frandsen, M. Kristensen, J. S. Jensen, P. Shi, and O. Sigmund, "Topology optimization and fabrication of photonic crystal structures," Opt. Express 12, 1996-2001 (2004).

17. O. Sigmund, "Design of multiphysics actuators using topology optimization-Part I: One-material Structures," Comput. Methods Appl. Mech. Eng. 190, 6577-6604 (2001).

18. U. D. Larsen, O. Sigmund, and S. Bouwstra, "Design and fabrication of compliant mechanisms and material structures with negative Poisson's ratio," J. Microelectromech. Syst. 6, 99-106 (1997).

19. O. Sigmund and S. Torquato, "Composites with extremal thermal expansion coefficients," Appl. Phys. Lett. 69, 32033205 (1996).

20. M. Koshiba, Y. Tsuji, and S. Sasaki, "High-performance absorbing boundary conditions for photonic crystal waveguide simulations," IEEE Microw. Wireless Compon. Lett. 11, 152-154 (2001).

21. J. S. Jensen and O. Sigmund, "Topology optimization of photonic crystal structures: a high-bandwidth low-loss T-junction waveguide," J. Opt. Soc. Am. B 22, 1191-1198 (2005).

22. O. Sigmund, "Morphology-based black and white filters for topology optimization," Struct. Multidiscip. Optim. 33, 401424 (2007).

23. J. K. Guest, "Topology optimization with multiple phase projection," Comput. Methods Appl. Mech. Eng. 199, 123135 (2009).

24. K. Svanberg, "A class of globally convergent optimization methods based on conservative convex separable approximations," SIAM J. Optim. 12, 555-573 (2002).

25. EURYI, http://www.esf.org/euryi.

26. TopAnt, http://www.topant.dtu.dk.

27. http://www.topopt.dtu.dk. 